\title{
A Color Interpolation Method for Bayer Filter Array Images Based on Direction Flag
}

\author{
Zheng Liu, Huachuang Wang \\ Institute of Optics and Electronics Chinese Academy of Sciences, \\ Sichuan Province, 610209, China
}

\begin{abstract}
The paper presents a color interpolation method for Bayer filter array images based on direction flag. The proposed algorithm determines four edge patterns defined through direction flag by four nearest green values surrounding the green interpolation location. Firstly, Bayer differences between multichannel pixels in horizontal and vertical directions of the color filter interpolation case are obtained. Secondly according to direction flag and edge pattern of the center pixel, missing green components of the center pixel are interpolated by different formulas in the horizontal, vertical and diagonal directions. Finally, correlation among channels is adopted to reconstruct remaining red/blue components pixels. Experimental results show that the presented method for Bayer CFA images based on direction flag can provide better reconstruction quality qualitatively and quantitatively.
\end{abstract}

Keywords-color filter array (CFA); demosaicking; direction flag; SSIM.

\section{INTRODUCTION}

Color interpolation is a basic arithmetic which is widely used in the image processing field. Considering that most color imaging equipments such as digital cameras capturing color images with a single CCD chip or CMOS sensor with a Color Filter Array (CFA) on the photosensitive surface to minimize the cost and packing size. Each pixel in the captured image can only obtain one component of the physical primary three colors (red, green, blue). The Bayer color filter array [1] with a mosaic pattern shown in Fig. 1 is commonly utilized.

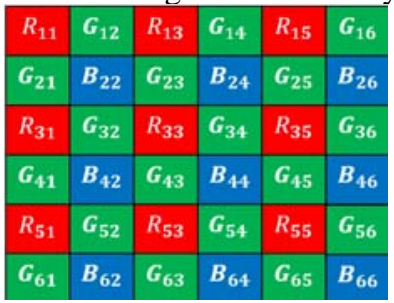

Fig.1. Bayer color filter array

In order to get the full color image, surrounding pixels are used to recover the other two missing color components at each pixel by color interpolation. There have been a large number of interpolation algorithms [2 8] over the past few decades, which aimed to reconstruct the missing colors as closely to the original ones as possible. Traditional interpolation algorithms mainly include two categories. The first is independent interpolation algorithm using a single color channel, including the neighborhood interpolation method, bilinear interpolation and cubic convolution interpolation method [2]. The simplest and most referenced one is probably bilinear interpolation, which uses the same color component linear averaging in $3 \times 3$ Bayer pattern block to estimate a pixel value. However, it introduces many color artifacts in the edge regions that blur the resulting image. The second is correlation interpolation method by interpolating the color differences between green $(G)$ pixel and red/blue $(\mathrm{R} / \mathrm{B})$ pixel, including edge gradient interpolation method $[3,4]$, adaptive interpolation method $[5,6]$ and so on. Laroche [4] improved an effective color interpolation method with edge direction detection by nearest green values surrounding the green interpolation location. It takes no account of the fact that the color difference pixels are not as uniform as assumed. Hamilton and Adams [5] reasonably improved color interpolation method that Laplacian second-order correction terms and gradient values in the horizontal and vertical directions are adopted as indicators, which can greatly reduce color artifacts along image edges. However, some resulting images across edges may contain zipper effects and color shifts during interpolation.

By studying the characteristics of traditional typical interpolation algorithms for Bayer filter array image, this paper presents a color interpolation method based on direction flag. In this algorithm, the relation of each color channel is taken into account and gradient operators in the horizontal, vertical and diagonal directions are used to compute the weighted values, which properly correct edges and details of the image. The efficient reconstruction in green channel helps to improve the restoration quality for red/blue component.

Experimental results show that an ideal visual effect of the reconstructed image with higher object SSIM value is achieved. This algorithm resolves effectively the conflicts between reconstructing high quality color image and reducing computational complexity, and thus largely enhances the edge and details quality.

\section{THE PROPOSED INTERPOLATION ALGORITHM FRAME}

The proposed algorithm firstly reconstructs green component images by estimating all the missing green values in Bayer CFA pattern image and determines the edge pattern of the center pixel by four direction gradient operators surrounding the green interpolation location. Secondly the direction flag of each center pixel is defined by the edge pattern. Meanwhile, pixel differences surrounding the green interpolation location between 
multichannel in horizontal and vertical directions are obtained parallelly. Thirdly, according to direction flag and edge pattern of the center pixel, missing green components are interpolated by different formulas in the horizontal, vertical and diagonal directions. Finally, multichannel correlation is adopted to reconstruct remaining red/blue component pixels. The frame chart of the proposed algorithm is shown in Fig. 2.

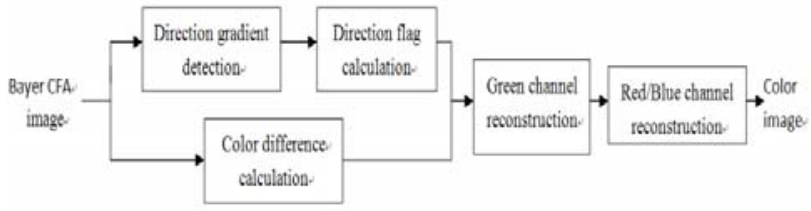

Fig.2. Frame chart of our interpolation algorithm

\section{A. Estimation of missing green values}

Considering the correlation of multichannel color components and reducing the computational complexity, we take only one color component gradient into account while calculating direction flag. Direction flag is defined as follows: the edge pattern is level if flag $=00$, the edge pattern is vertical if flag $=01$, the edge pattern is diagonal $45^{\circ}$ or $-135^{\circ}$ if flag $=10$, the edge pattern is diagonal $135^{\circ}$ or $-45^{\circ}$ if flag $=11$.

All missing green values are estimated by combining edge detection interpolation method with weighted coefficients method. Only adjacent green pixels are involved in the edge detection operator which is shown in Fig. 3. The detailed operation is described as follows:

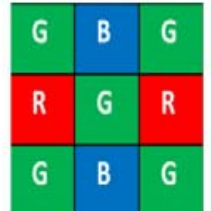

(a).

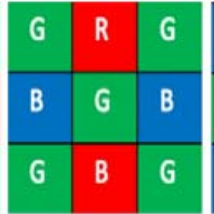

(b).

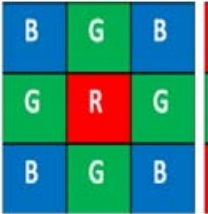

(c)

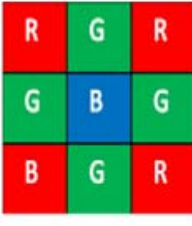

(d) .
Fig.3. Four Bayer pattern array

Defining the level gradient operator $\alpha$,vertical gradient operator $\beta$,diagonal gradient operator $\varphi$ and $\omega$ in center pixel location $(i, j)$.

As in shown in Fig. 3, calculating gradient operator for the (a) (b) pattern according to Eq. 1:

$$
\begin{aligned}
\alpha & =\frac{1}{2} *|G(i-1, j+1)+G(i+1, j+1)-G(i-1, j-1)-G(i+1, j-1)|(1) \\
\beta & =\frac{1}{2} *|G(i-1, j-1)+G(i-1, j+1)-G(i+1, j-1)-G(i+1, j+1)| \\
\varphi & =\frac{1}{2} *|G(i-1, j-1)+G(i-1, j+1)-G(i+1, j-1)-G(i-1, j+1)| \\
\omega & =\frac{1}{2} *|G(i-1, j-1)+G(i+1, j+1)-G(i+1, j-1)-G(i-1, j+1)|
\end{aligned}
$$

Meanwhile calculating pixel differences of green with red/blue channel in horizontal and vertical directions according to Eq. 2:

$$
\begin{aligned}
& K_{g r}(i, j)=G(i, j)-\frac{R(i, j-1)+R(i, j+1)}{2} \\
& K_{g b}(i, j)=G(i, j)-\frac{B(i, j-1)+B(i, j+1)}{2}
\end{aligned}
$$

Calculating gradient operator for the (c) (d) pattern according to Eq. 3:

$$
\begin{aligned}
\alpha & =|G(i, j+1)-G(i, j-1)| \\
\beta & =|G(i-1, j)-G(i+1, j)| \\
\varphi & =\frac{1}{2} *|G(i, j+1)+G(i, j-1)-G(i+1, j)-G(i, j-1)| \\
\omega & =\frac{1}{2} *|G(i, j-1)+G(i-1, j)-G(i+1, j)-G(i, j+1)|
\end{aligned}
$$

After obtaining the direction gradient operators, direction flag values are defined as the minimum of all direction gradient operators shown in Eq. 4.

$$
\text { flag }= \begin{cases}00 & \text { if } \min (\alpha, \beta, \varphi, \omega)=\alpha \\ 01 & \text { if } \min (\alpha, \beta, \varphi, \omega)=\beta \\ 10 & \text { if } \min (\alpha, \beta, \varphi, \omega)=\varphi \\ 11 & \text { if } \min (\alpha, \beta, \varphi, \omega)=\omega\end{cases}
$$

Missing green components are estimated based on direction flag values and weighted coefficients of difference of green with red/blue channel and four nearest green values surrounding the green interpolation location. The detailed algorithm is described in Eq. 5:

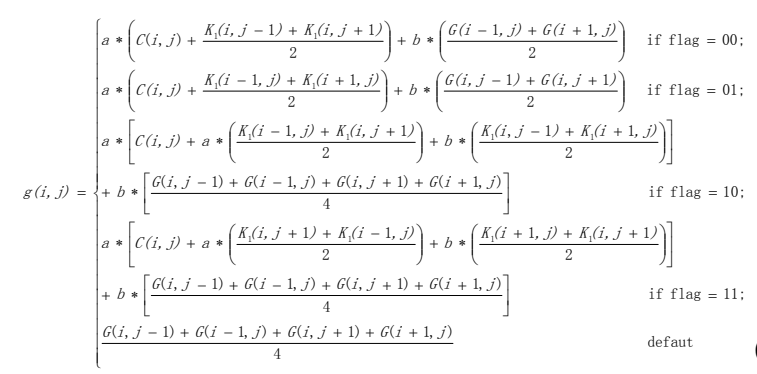

For the (c) pattern, $C(i, j)=R(i, j), K_{1}=K_{g r}$,for the (d) pattern, $C(i, j)=B(i, j), K_{1}=K_{g b}$.

\section{B. Red and blue channel interpolation}

After estimating the whole green components, for simplicity, we substitute the high frequency components of $R$ and $B$ channels with those of the $G$ channel, and then the $\mathrm{R}$ and $\mathrm{B}$ values can be interpolated by employing the color difference rule, i.e. $\Delta R=G-R, \Delta B=G-B$.

For the (a) (b) pattern, location of the center pixel is defined as $(i, j)$, missing red and blue components are obtained according to Eq. 6: 


$$
\begin{aligned}
& r(i, j)=\frac{P 1+P 2}{2}-\frac{G(i, j-1)+G(i, j+1)}{2}+G(i, j) \\
& b(i, j)=\frac{Q 1+Q 2}{2}-\frac{G(i-1, j)+G(i+1, j)}{2}+G(i, j)
\end{aligned}
$$

In the (a) and (b) pattern respectively calculated in Eq. 7:

$$
\begin{array}{llll}
P 1=R(i, j-1) & P 2=R(i, j+1) ; & Q 1=B(i-1, j) & Q 2=B(i+1, j) \\
P 1=R(i-1, j) & P 2=R(i+1, j) ; & Q 1=B(i, j-1) & Q 2=B(i, j+1)
\end{array}
$$

For the (c) (d) pattern, missing red or blue components are obtained according to Eq. 8:

$$
\begin{aligned}
b(i, j)= & G(i, j)+\frac{B(i-1, j-1)+B(i-1, j+1)+B(i+1, j-1)+B(i+1, j+1)}{4} \\
& -\frac{G(i-1, j-1)+G(i-1, j+1)+G(i+1, j-1)+G(i+1, j+1)}{4} \\
r(i, j)= & G(i, j)+\frac{R(i-1, j-1)+R(i-1, j+1)+R(i+1, j-1)+R(i+1, j+1)}{4} \\
& -\frac{G(i-1, j-1)+G(i-1, j+1)+G(i+1, j-1)+G(i+1, j+1)}{4}
\end{aligned}
$$

In this way, all the missing color components in the Bayer filter array images have been estimated by above interpolation.

\section{EXPERIMENTAL RESULTS AND ANALYSIS}

The purpose of color interpolation experiment is to test the processing ability for color filter array images, including reconstructed image quality and processing time. Subsequently, we shall test the performance of the proposed algorithm through both objective visual and subjective quality.

The aim of this experiment is to compare some traditional interpolation algorithms with the proposed algorithm in term of visual effect. To reduce the error caused by subjective factors, we choose a series of source images from LIVE standard test image library provided by University of Texas at Austin. The following traditional interpolation algorithms are taken as comparison: Nearest interpolation, Bilinear interpolation, (Cok) constant hue interpolation [7], (Laroche) edgedetected Interpolation, (Hamilton) adaptive interpolation.

Taking the image $(768 \times 512)$ "Parrots", $(480 \times 720)$ "Womanhat" for illustration, the comparison of reconstructed images with the use of different methods is shown in Fig. 4, Fig. 5. For the convenient of comparison and observation, a local zoom window is presented in the upper right corner of the display image. It seems that there is no difference between the reconstructed visual images and source images. Despite that we still can find that color artifacts and zipper effects along image edges in comparison of local zoom windows, the reconstructed image quality interpolated by the proposed method performs a better visual effect with less false colors and edge distortion. The reason lies in that the method takes multichannel correlation into consideration as well as adding more edge detection directions when estimating the missing green channel.
An objective quality indicator is SSIM. Structural similarity theory proposed by Zhou Wang [9] respectively compares the brightness, contrast and structural properties between two images and then products weighted value. SSIM is used as a measure of quality, as expressed in Eq. 9. Where $u_{x}$ and $u_{y}$ are the linear averaging of the image in the direction of $x$ and $y$, and where and $\sigma_{y}$ are the gray differences of the image in the direction of $X$ and $y$. The closer with 1 value of SSIM is, the more similar both images are. In other words, the closer with 1 value of SSIM means the better quality the interpolated image reconstructs. Thus, the resulting interpolated pixel values were compared with the original images in terms of SSIM. Table 1 shows the data results of comparing

\begin{tabular}{|c|c|c|c|c|}
\hline \multirow{4}{*}{ images } & \multicolumn{3}{|c|}{ Nearest } & \multirow[t]{2}{*}{ Bilinear } \\
\hline & Cok & & & \\
\hline & SSIM & Time(s) & SSIM & Time(s) \\
\hline & SSIM & Time(s) & & \\
\hline \multirow{12}{*}{$\begin{array}{l}\text { Caps } \\
\text { Bikes } \\
\text { Parrots } \\
\text { Womanhat } \\
\text { Plane } \\
\text { Cemetry }\end{array}$} & 0.9409 & 0.353981 & 0.9649 & 0.421587 \\
\hline & 0.9697 & 0.992636 & & \\
\hline & 0.8892 & 0.360260 & 0.9470 & 0.418929 \\
\hline & 0.9690 & 1.019446 & & \\
\hline & 0.9596 & 0.357149 & 0.9778 & 0.429015 \\
\hline & 0.9760 & 1.005412 & & \\
\hline & 0.9486 & 0.262068 & 0.9672 & 0.318709 \\
\hline & 0.9677 & 0.751466 & & \\
\hline & 0.9291 & 0.376640 & 0.9557 & 0.427630 \\
\hline & 0.9651 & 1.025592 & & \\
\hline & 0.8826 & 0.240989 & 0.9461 & 0.292989 \\
\hline & 0.9702 & 0.681825 & & \\
\hline \multirow{4}{*}{ images } & & Laroche & & Hamilton \\
\hline & Proposed & & & \\
\hline & SSIM & Time(s) & SSIM & Time(s) \\
\hline & SSIM & Time(s) & & \\
\hline \multirow{12}{*}{$\begin{array}{l}\text { Caps } \\
\text { Bikes } \\
\text { Parrots } \\
\text { Womanhat } \\
\text { Plane } \\
\text { Cemetry }\end{array}$} & 0.9751 & 0.896064 & 0.9772 & 1.023895 \\
\hline & 0.9843 & 1.469000 & & \\
\hline & 0.9722 & 0.894698 & 0.9736 & 0.998962 \\
\hline & 0.9795 & 1.476813 & & \\
\hline & 0.9825 & 0.899057 & 0.9829 & 1.014488 \\
\hline & 0.9836 & 1.469494 & & \\
\hline & 0.9724 & 0.688428 & 0.9727 & 0.790453 \\
\hline & 0.9733 & 1.134200 & & \\
\hline & 0.9667 & 0.922624 & 0.9680 & 1.106603 \\
\hline & 0.9708 & 1.467800 & & \\
\hline & 0.9720 & 0.603062 & 0.9741 & 0.682201 \\
\hline & 0.9752 & 0.992821 & & \\
\hline
\end{tabular}
source tested images with reconstructed full color images interpolated by six demosaicking methods in terms of the SSIM value and processing time.

$$
\operatorname{SSIM}(x, y)=\frac{\left(2 u_{x} u_{y}+c_{1}\right)\left(2 \sigma_{x} \sigma_{y}+c_{2}\right)}{\left(u_{x}^{2}+u_{y}^{2}+c_{1}\right)\left(\sigma_{x}^{2}+\sigma_{y}^{2}+c_{2}\right)} \text {. }
$$

TABLE 1. SSIM AND TIME COMPARISON FOR DIFFERENT INTERPOLATED COLOR IMAGES algorithm performs a higher SSIM values than all of the 
other listed methods without increasing much more time. Comparing all five demosaicing algorithms with the proposed interpolation method, we can find that the proposed algorithm makes a good balance between the computational complexity and the objective SSIM value, and experimental results are satisfactory.

\section{CONCLUSIONS}

In this paper, we propose a color interpolation method for Bayer filter array images based on detection flag. Testing a serials of LIVE standard images by taking multichannel correlation into consideration as well as adding more reasonable edge detection directions when estimating the missing color components, the proposed interpolation method has been proved that an ideal visual effect of the reconstructed image with higher objective SSIM value could be achieved. Meanwhile, it makes a good balance between computational complexity and quality of image after interpolation.

However, it should also be noted that the tested images are so ideal without considering interference from inner noises. Moreover, the algorithm will be more efficient if the direction flag is used in the process of interpolating the missing red/blue channel. Thus, much more work is needed for different images and conditions. In conclusion, due to its low complexity and good interpolation performance, the new proposed method is suitable for Bayer CFA images demosaicking.

\section{REFERENCES}

[1] Bayer B E. Color imaging array US Patent: 3971065[P]. 1976-07-27.

[2] Robert K. Cubic convolution interpolation for digital image processing [J]. IEEE Trans. Acoust.Speech. Signal Processing. 1981,29(6): 1153-1160

[3] R.H. Hibbard. Apparatus and method for adaptively interpolating a full color image utilizing luminance gradients. U.S. Patent, No.5382976, 1995.

[4] C.A. Laroche, M.A. Prescott. Apparatus and method for adaptively interpolating a full color image utilizing chrominance gradients. U.S. Patent, No.5373322, 1994.

[5] J.F. Hamilton, J.E. Adams. Adaptive color plane interpolation in single sensor color electronic camera. U.S. Patent, No.5629734, 1997.

[6] J.E. Adams. Design of practical color filter array interpolation algorithms for digital cameras. Proc. SPIE, Real Time Imaging. 1997, Vol.3028:117-125.

[7] D.R. Cok. Signal processing method and apparatus for producing interpolated chrominance values in a sampled color image signal. U.S. Patent, No.4642678, 1987.

[8] Ron Kimmel. Demosaicing: Image reconstruction from color CCD samples. IEEE Transactions on Image Processing. 1999, Vol.8, NO.9:1221-1228.

[9] Wang, Z., Bovik, A.C., Sheikh, H.R., et al. Image quality assessment: from error visibility to structural similarity [J].IEEE Trans. Image Process, 2004, 13(4):600-612. 

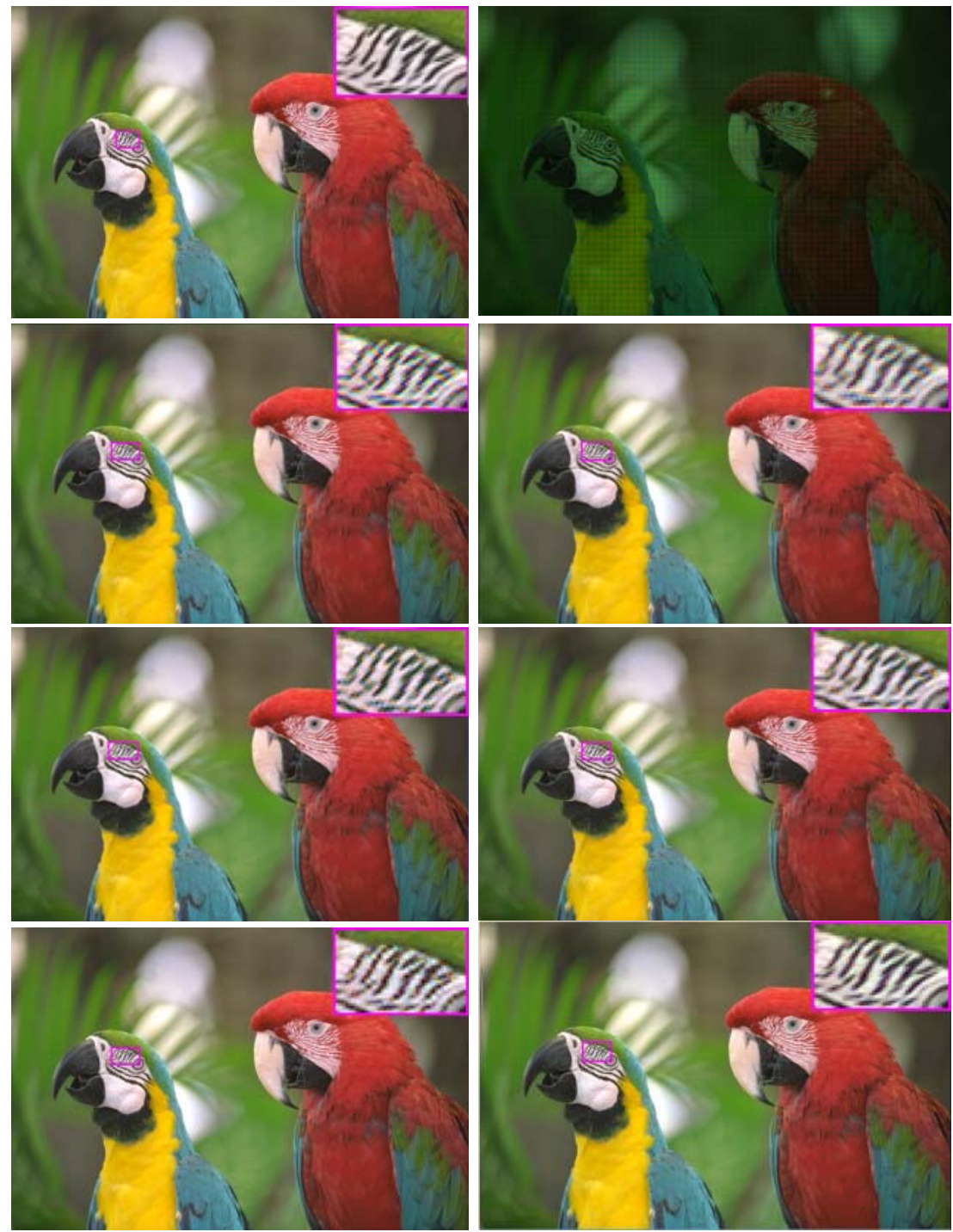

Fig.4. comparison of the Original and demosaicked results of parrots. From the first image to the last image: Original image, Bayer image, Nearest image, Bilinear image, Cok image, Laroche image, Hamilton image, Proposed image. 

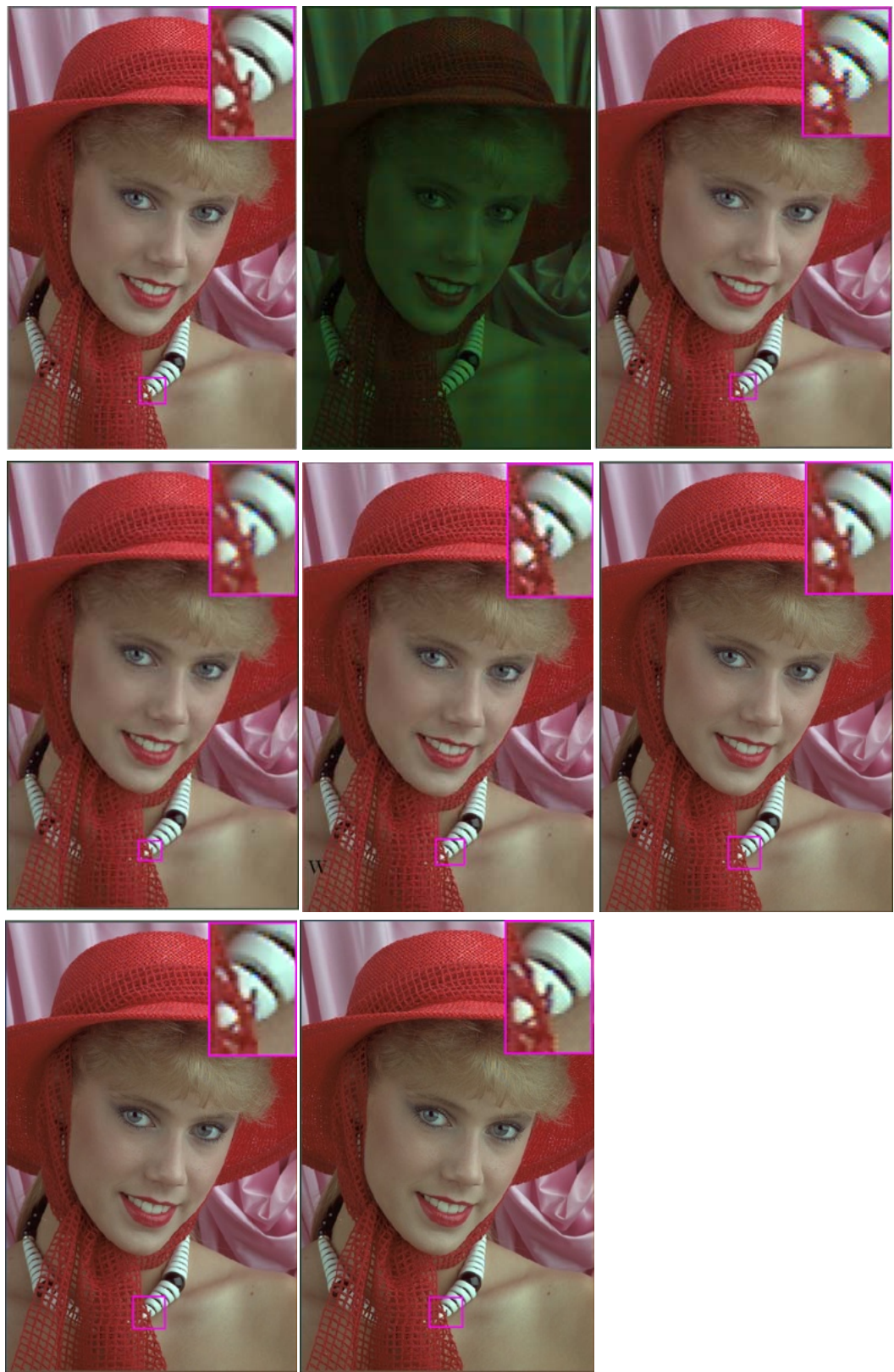

Fig.5. comparison of the Original and demosaicked results of womanhat. From the first image to the last image: Original image, Bayer image, Nearest image, Bilinear image, Cok image, Laroche image, Hamilton image, Proposed image. 